\title{
Soy may help protect against cardiovascular disease
}

\author{
by Emily R. Cena and Francene M. Steinberg
}

\section{Diet and lifestyle choices are major} factors contributing to the risk of cardiovascular disease, which is responsible for more deaths in the United States than any other cause. One dietary component that has received considerable attention for its potential cardioprotective effects is soybeans, which contain lean vegetable protein, dietary fiber and bioactive compounds known as isoflavones. Recent research investigating the relationship between soy and cardiovascular disease has identified several potential mechanisms for the observed protective effects, including cholesterol-lowering properties, antioxidant activity and gene regulation. This review highlights current understanding of the complex relationship between soy and the risk of cardiovascular disease.

ardiovascular disease - comprised of heart disease, heart failure and stroke - is the leading cause of death in the United States. The estimated combined costs of health care services, medications and lost productivity attributed to cardiovascular disease were more than $\$ 475$ billion in 2009 (Lloyd-Jones et al. 2009).

Although this chronic inflammatory disease affects the lives of millions of Americans, the development of cardiovascular disease is somewhat preventable. Since diet is arguably the most modifiable risk factor, scientists have devoted a great deal of research to the relationship between dietary choices and cardiovascular disease. A healthy diet is generally high in fruits, vegetables, whole grains and legumes. These plant foods tend to be rich in bioactive compounds, or "extra-nutritional" constituents, that are associated with reducing the risk of cardiovascular disease.

One dietary component of considerable research interest is soybeans, a legume

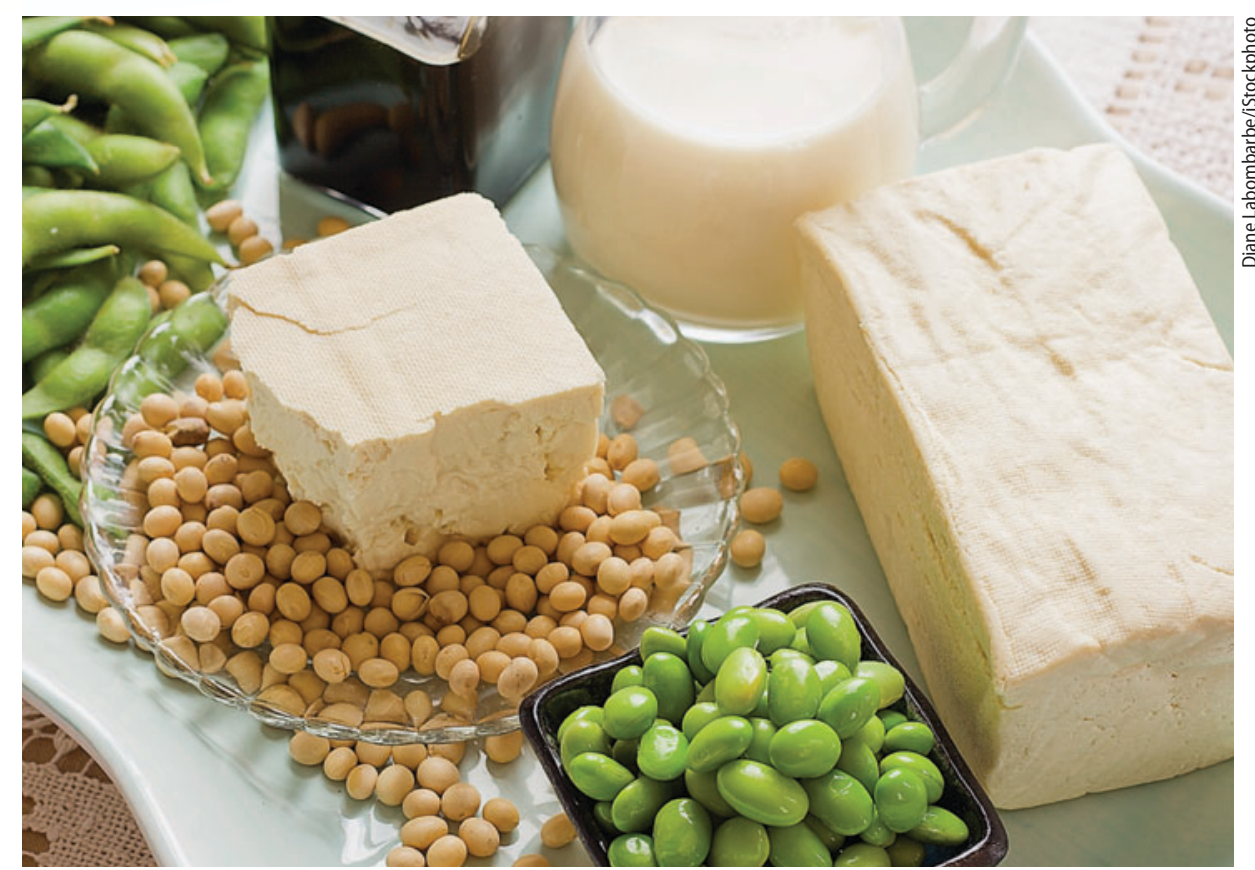

Soybeans and foods made from soy are the major source of isoflavones, which serve as antioxidants, scavenging and neutralizing free radicals that might otherwise cause inflammation and increase the risk of heart disease.

linked to the decreased risk of cardiovascular disease. Epidemiological studies suggest that Asian populations consuming large amounts of soy have lower rates of cardiovascular disease than Western populations (Zhang et al. 2003). In the mid-1990s, a meta-analysis of 29 clinical trials found that compared to animal protein, soy protein significantly reduced blood levels of several lipids (total cholesterol, LDL cholesterol and triglycerides) (Anderson et al. 1995). This prompted the U.S. Food and Drug Administration to approve the current health claim that 25 grams of soy protein per day, as part of a diet low in saturated fat and cholesterol, may reduce the risk of heart disease.

While an American Heart Association statement issued in 2006 supported the inclusion of soy in the diet, it was not definitive about the protective mechanism of action (Lichtenstein et al. 2006). Research over the past 10 years has reported mixed results due to varying study designs and soy preparations, but the literature overall still supports the conclusion that soy protein with isoflavones can decrease blood levels of LDL cholesterol (Zhan and Ho
2005). Moreover, recent studies suggest that the relationship between soy and cardiovascular disease is more complex than just lowering cholesterol to protect against atherosclerosis.

\section{How soy isoflavones work}

Effects. Soybeans provide high-quality vegetable protein, dietary fiber and bioactive compounds called isoflavones, a class of phytochemicals (or plant compounds)

Isoflavones are found almost exclusively in soybeans and foods made from soy.

that provide health benefits. Many different phytochemicals have been identified in nature and studied for their health effects. For example, anthocyanins found in red, blue and purple fruits and vegetables (such as berries and grapes) have been associated with reduced inflammation and lower risk of heart disease. Sulforaphane, a phytochemical found in broccoli and

Online: http://californiaagriculture.ucanr.org/ landingpage.cfm?article=ca.v065n03p118\&fulltext=yes DOI: 10.3733/ca.v065n03p118 
other cruciferous vegetables, may reduce the risk of certain types of cancer.

Isoflavones are found almost exclusively in soybeans and foods made from soy. The cardiovascular health benefits associated with isoflavones appear to be due to the fact that in the body they serve as antioxidants, which neutralize the free radicals that cause oxidative damage to cells. A free radical is a molecule containing an unpaired electron, which makes it highly reactive. Within blood vessels, free radicals can oxidize circulating LDL cholesterol, starting a cascade of inflammatory events that ultimately increase the risk of developing heart disease. Antioxidants interfere with this process by neutralizing, or scavenging, the free radicals.

Likewise, soy acts like compounds similar to the hormone estrogen, affecting processes including blood vessel dilation and gene regulation (fig. 1, page 120). Some health concerns have been raised related to soy's estrogenlike properties, particularly with regard to breast cancer risk, but these concerns are beyond the scope of the current article. Soy protein has also been shown to provide health benefits, such as helping to reduce LDL cholesterol levels. In addition, eating intact soy protein and isoflavones may improve vascular function better than either component by itself (Steinberg et al. 2003).

Bioavailability. The amount of isoflavones in a person's diet does not directly determine their bioavailability, the amount of isoflavone metabolites that reach the body's tissues and so are available to exert physiological effects. Rather, bioavailability depends on absorption, metabolism, distribution and excretion (fig. 1) (Lampe and Chang 2007). The isoflavones in soy are not biologically active until after they are digested and absorbed in the small intestine. Enzymes in the small intestine remove glycoside, or sugar, residues from the isoflavones, turning them into bioactive forms that can be absorbed.

Metabolites. In humans, the primary forms of bioactive isoflavone are daidzein and genistein, which are similar to the hormone estrogen. Daidzein and genistein are absorbed along the length of the small intestine, where they undergo further metabolism. The resulting variety of isoflavone metabolites, such as glucuronides and sulphates, then leave the liver and enter the systemic circulation. These metabolites travel to various tissues of the body, where they exert their cellular effects.

\section{Isoflavone metabolism}

Some isoflavone metabolites are secreted by the liver into bile, which is then secreted into the small intestine. There, the isoflavone metabolites in the bile once again undergo digestion and absorption. This recycling process is known as enterohepatic (or intestine-liver) circulation.
Another type of recycling, known as enteric recycling, occurs in the small intestine, where isoflavones are metabolized into glucuronidated and sulphated forms that are then secreted back into the intestine for further reabsorption and metabolism (Chen et al. 2003).

Enterohepatic circulation and enteric recycling of isoflavones are important because they allow body tissues to be exposed to these phytochemicals for longer amounts of time following soy intake. After absorption, metabolism and distribution, isoflavones are ultimately

\section{Glossary}

Bioactive compounds: Any chemical compounds, organic or inorganic, that exert physiological effects on a living organism. Bioactive compounds generally refer to those found in foods or metabolites formed in the body that affect human health, such as soy isoflavones.

Cholesterol (HDL, LDL, total): A type of lipid that is used by the body for cell membrane structure, cell signaling and the synthesis of hormones, bile and vitamin D. Sources include synthesis by the liver and the dietary intake of animal products. Cholesterol is transported through the bloodstream bound to lipoproteins that are classified according to their lipid-to-protein ratio. Low-density lipoproteins (LDL) contain relatively large amounts of fat and cholesterol compared to protein, whereas high-density lipoproteins (HDL) contain more protein and less cholesterol. LDL cholesterol is often referred to as "bad" cholesterol because it can become oxidized by free radicals, resulting in inflammation and atherosclerosis. High levels of HDL cholesterol, sometimes called "good" cholesterol, are associated with lower risk of heart disease and other chronic diseases, possibly because HDL molecules transport cholesterol to the liver for excretion.

Cytokines: Intercellular signaling molecules involved in physiological processes such as inflammation. Cytokines consist of many different small molecules that enable cells to communicate. Some examples include tumor necrosis factor alpha (TNF- $\alpha$ ) and interleukins such as interleukin-6 (IL-6), both of which are involved in the inflammatory process.

Endothelial cells, function: Cells that line blood vessels throughout the body, providing a barrier between the bloodstream and body tissues. They are actively involved in blood clotting, controlling white blood cell migration in and out of the bloodstream during the inflammatory response, and constriction and dilation of blood vessels.

Estrogen: A primary sex hormone that performs a number of important functions related to human health. Although present at higher levels in women, estrogen helps regulate reproductive development and function in men and women. Estrogen reduces the risk of cardiovascular disease by decreasing LDL cholesterol and triglyceride levels, and increasing HDL cholesterol. Estrogen also plays a key role in bone health.

Triglyceride: The predominant form of fat in food and in the body, consisting of a glycerol backbone with three fatty acids bound to it. Most circulating fat and most fat stored in adipose cells is in the form of triglycerides. Elevated triglyceride levels are a marker of increased risk for heart disease, diabetes and other chronic diseases, and high triglycerides are one of the defining components of the metabolic syndrome. 
excreted in urine, and most excretion occurs within 24 hours of consuming a soy-containing meal (Setchell et al. 2003). Isoflavones that pass through the small intestine without being bioactivated then pass into the colon, where precursors to the bioactive form of daidzein are instead metabolized by bacteria into another bioactive compound called equol. The equol is then absorbed into the bloodstream and transported to various body tissues, where it appears to exhibit greater antioxidant and estrogenic effects than daidzein (Arora et al. 1998; Mitchell et al. 1998). Although there are other bacterial byproducts of isoflavone metabolism in the colon, equol is the primary one with significant bioactivity in humans.

Not all humans have colonic bacteria capable of producing equol. Equol is produced by an estimated $30 \%$ to $40 \%$ of white persons (Lampe et al. 1998) and up to $60 \%$ of Asians (Morton et al. 2002). Factors associated with equol production are not fully understood but are believed to include genetics, diet and lifestyle, in addition to the composition of gut microflora (Atkinson et al. 2008). It is unclear whether equol producers have increased protection against cardiovascular disease compared to nonproducers, and this remains a topic of great research interest (Atkinson et al. 2005).

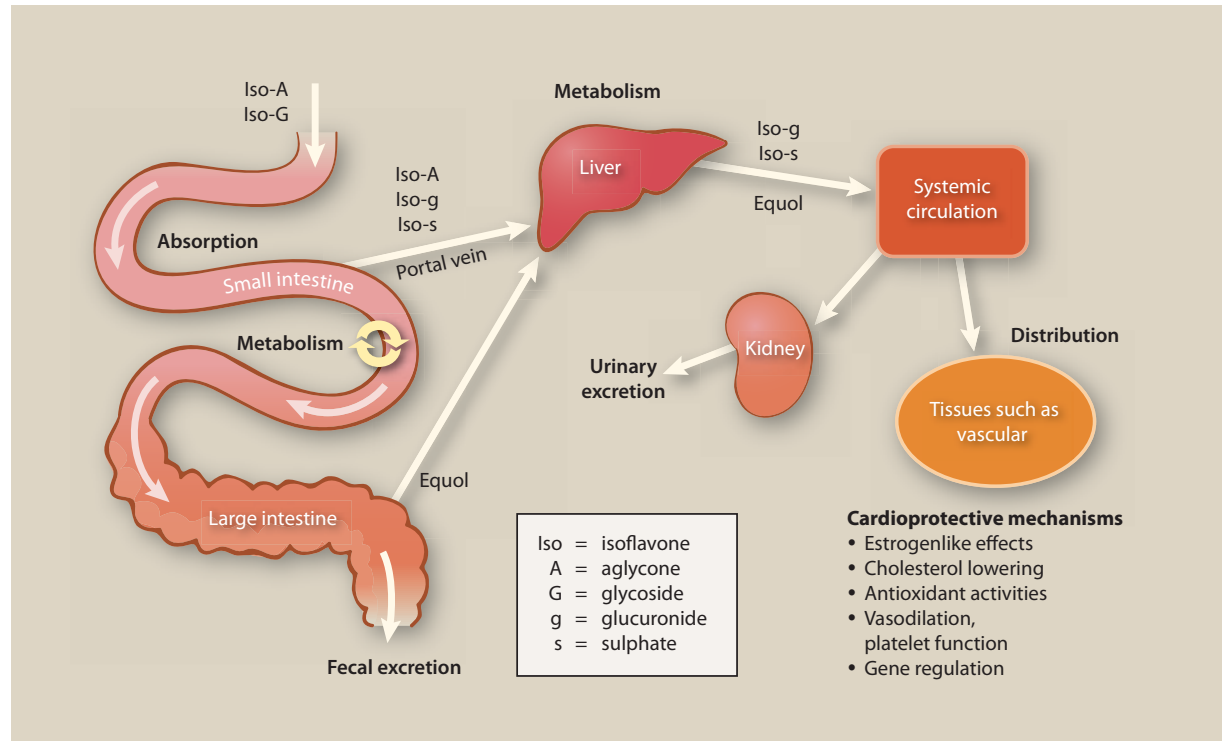

Fig. 1. Overview of the absorption, metabolism, distribution and excretion of soy isoflavone metabolites.

\section{Cardiovascular disease protection}

Atherosclerosis, which hardens and narrows the arteries, is a primary cause of cardiovascular disease (see box). The development of atherosclerosis is quite complex, involving chronic inflammation, the generation of reactive oxygen species, abnormal endothelial cell function, lipid-laden foam cell formation and clot formation within blood vessels. The observed protective effect of soy and soy

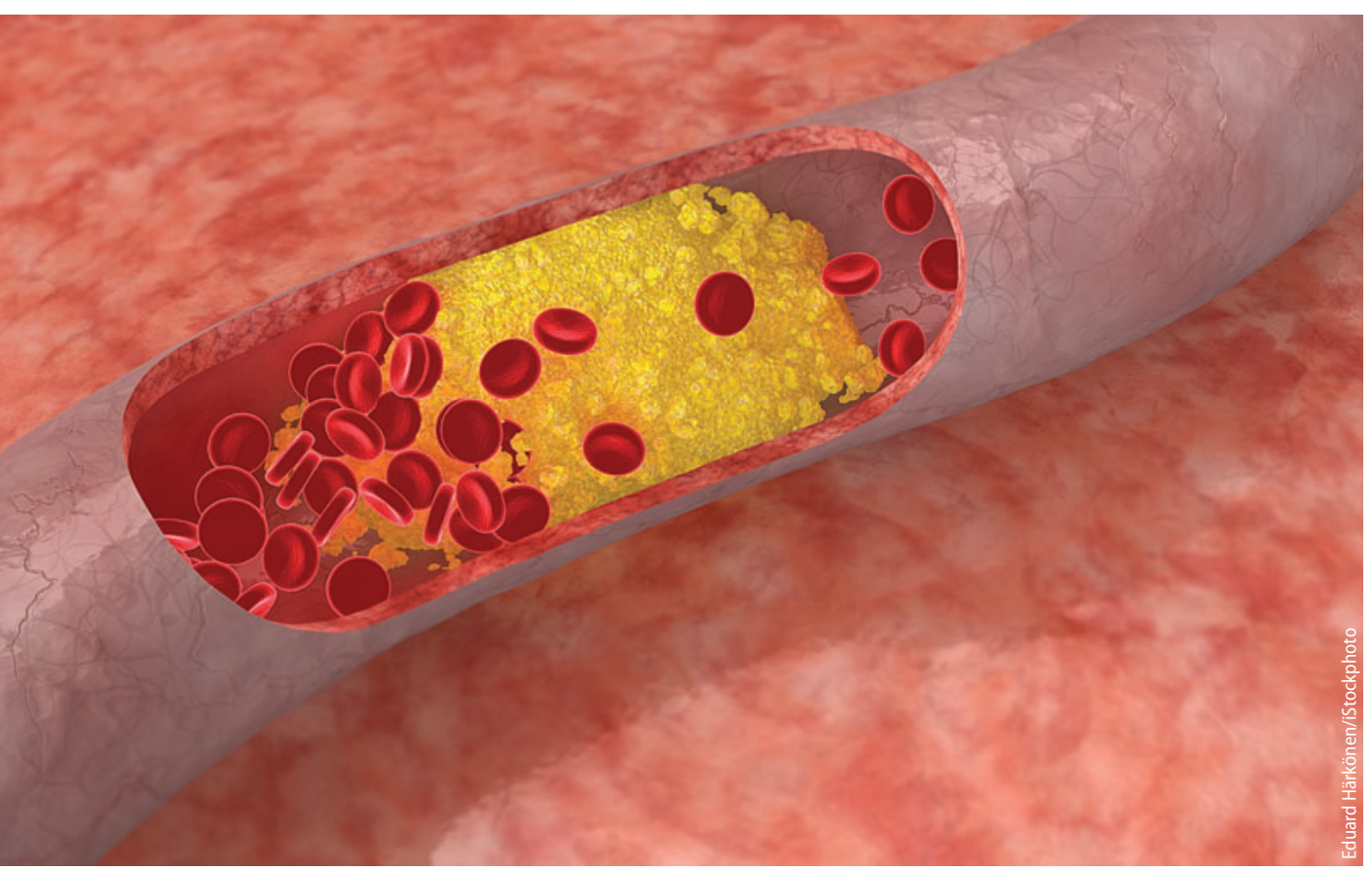

Soy can reduce LDL cholesterol circulating in the blood, thereby decreasing the formation of plaque in blood vessel walls. isoflavones on atherosclerosis and cardiovascular disease risk appears to be related to a number of different mechanisms.

Estrogenlike effects. In cardiovascular disease, the blood vessels become constricted or blocked, forcing the heart to work harder to push blood through the body. One factor that promotes blood vessel dilation (vasodilation) is the production of nitric oxide by endothelial cells in the blood vessel wall. Estrogen can rapidly increase endothelial nitric oxide production (Rimbach et al. 2008), providing anti-atherosclerotic effects. Because isoflavones are so similar to estrogens, they can also rapidly increase endothelial nitric oxide production, enhancing vasodilation and improving blood flow.

Another factor contributing to the development of cardiovascular disease is inflammation in blood vessels. In animal models, estrogen decreases the levels of two small proteins (cytokines TNF- $\alpha$ and IL-6) that increase inflammation, reducing the risk of cardiovascular disease (Ito et al. 2001). It is unclear whether isoflavones similarly inhibit the production of proinflammatory cytokines in humans (Beavers et al. 2009), but this remains a current area of research interest.

Cholesterol-lowering properties. In addition to the widely recognized cholesterol-lowering effects of soy protein, soy isoflavones also help lower both total and LDL cholesterol levels, while increasing cardioprotective HDL cholesterol (Taku et al. 2007; Zhan and Ho 2005). In fact, 
the combination of soy protein and isoflavones appears to exhibit the strongest hypocholesterolemic effects compared to isolated soy protein or soy isoflavones alone (Mortensen et al. 2009). Whole soy can promote a $3 \%$ to $5 \%$ reduction in blood cholesterol (Lichtenstein et al. 2006; Zhan and Ho 2005). Although soy's effects are not as large as the $20 \%$ or greater reductions observed with statin drug therapy (Rosenson 2006), even a 3\% to 5\% reduction can have clinical significance. Reduced circulating LDL cholesterol means less is available to become oxidized and retained in lipid plaques in the blood vessel wall. Less oxidized LDL cholesterol results in a lower risk of atherosclerosis and therefore cardiovascular disease.

Antioxidant activity. Another way to reduce LDL cholesterol oxidation, aside from decreasing the amount that is circulating, is to prevent or slow oxidation through the action of antioxidants. Soy isoflavones and their metabolites, particularly genistein and equol, have antioxidant properties.

Two studies in humans demonstrated that daily consumption of soy protein with isoflavones reduced levels of LDL

\section{The risks of cardiovascular disease}

In 2006, cardiovascular disease was responsible for $26 \%$ of all deaths in the United States (Heron et al. 2009). In California alone, it claimed the lives of more than 73,000 people in 2004 - more than cancer, diabetes, chronic liver disease, homicide, suicide and HIV/AIDS combined (Reynen et al. 2007). The costs associated with the treatment of cardiovascular disease are staggering, particularly at a time when health care costs in the United States have never been higher.

Several risk factors for cardiovascular disease are at least partially modifiable by lifestyle choices, including obesity, high blood pressure, diabetes, chronic inflammation, dyslipidemia (abnormally high blood cholesterol and/or triglycerides), smoking, physical inactivity and dietary choices.

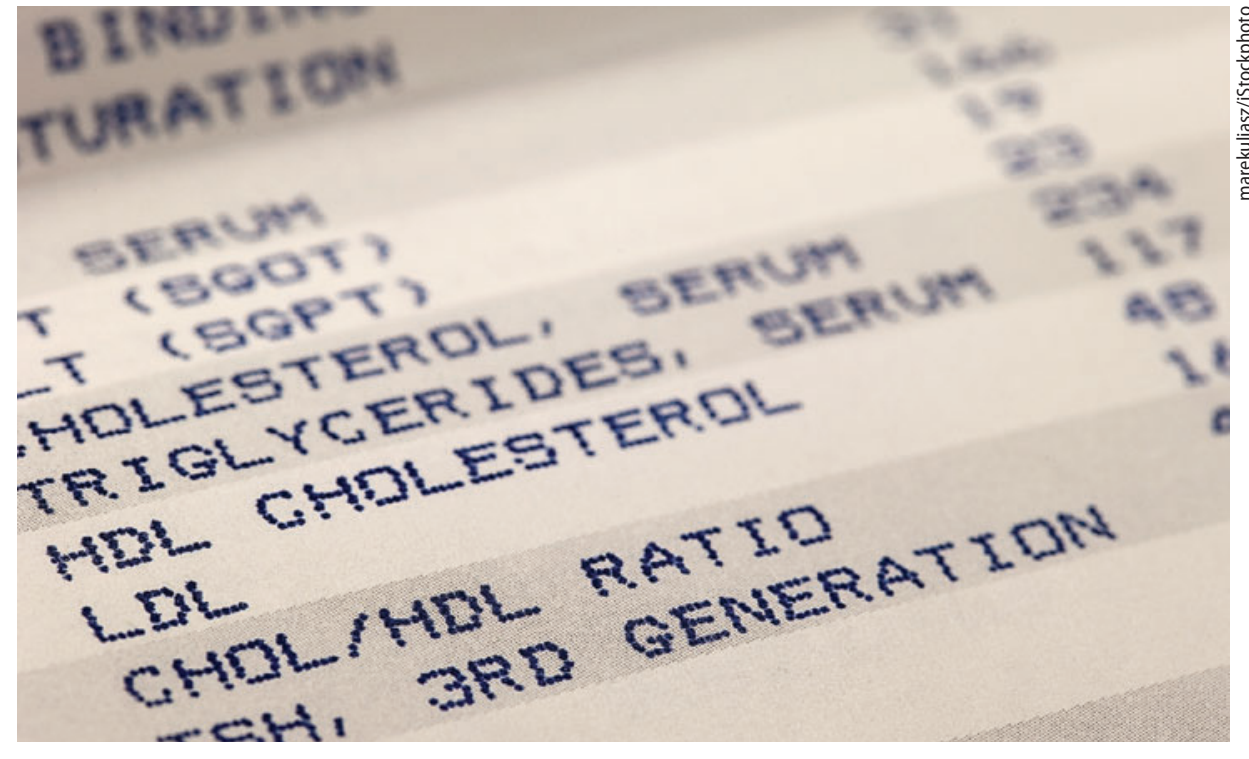

Soy isoflavones increase the levels of cardioprotective HDL ("good") cholesterol and decrease levels of LDL ("bad") cholesterol, lowering the risk of heart disease.

oxidation (Tikkanen et al. 1998; Wiseman et al. 2000). In vitro studies have revealed potential mechanisms for the antioxidant activity of isoflavones, including: (1) their ability to scavenge free radicals and chelate metals (or bind to metals, making them less chemically reactive); (2) their role in inhibiting the production of hydrogen peroxide, a naturally occurring cellular byproduct that oxidizes compounds and damages blood vessel walls, stressing the body by inflammation; and (3) their capacity to stimulate the production of antioxidant enzymes such as superoxide dismutase (Mortensen et al. 2009).

Reactive oxygen species are molecules, ions or free radicals that contain oxygen and are highly reactive because of unpaired electrons. Cellular processes produce them naturally, but large amounts cause oxidative damage. A key partner in cellular defenses against oxidative damage is the transcription factor Nrf2, which promotes the transcription of genes for various antioxidant and detoxifying enzymes. The activation of antioxidant genes via the Nrf2 pathway is a potential role for isoflavones (Mann et al. 2009).

Soy isoflavones help promote vasodilation by stimulating nitric oxide production in the endothelial cells lining blood vessels. Interestingly, macrophages (a type of white blood cell) also produce nitric oxide during the inflammatory response, in an effort to destroy foreign particles such as bacteria. In cases of chronic inflammation, however, increased nitric oxide production by macrophages causes oxidative stress that contributes to the formation of atherosclerotic plaques, thereby increasing the risk of cardiovascular disease. Soy isoflavones are able to inhibit inducible nitric oxide production in macrophages, reducing excessive oxidative stress (Rimbach et al. 2008).

Improved vasodilation. Soy isoflavones can promote vasodilation by stimulating the production of nitric oxide. A recent study showed that genistein, one of the estrogenlike forms of isoflavones, modified the expression of genes that encode for a number of proteins related to vasodilation and blood pressure (Ambra et al. 2006). While in vitro and animal studies seem to support the vasodilatory effects of isoflavones, a review of human intervention studies found conflicting results, with some studies reporting improved vasodilation and others reporting no change (Mortensen et al. 2009). In addition, isoflavones appear to promote vasodilation in ways that do not involve estrogenic effects. More research is necessary to better elucidate the relationship between isoflavones and vasodilation in humans.

Inhibition of platelet aggregation. One of the greatest threats of cardiovascular disease is the formation of clots within blood vessels. If a blood clot forms and breaks loose, it can travel to a coronary artery (which supplies the heart with blood), block the artery and cause a lifethreatening heart attack. Similarly, if a 
soy foods on biomarkers of cardiovascular risk in individuals with metabolic syndrome; it may be completed in 2012.

The cardioprotective effects of soy appear to be mediated by several mechanisms related to the inflammatory processes of atherosclerosis, involving both genomic and nongenomic activities. Soy isoflavones have a broad variety of biological actions. The beneficial effects of soy are modest compared to pharmacological treatment, such as statin drugs. However, the cumulative effects over a lifetime are likely to be significant. Soy and its bioactive isoflavone components are an important part of a diet rich in foods for health promotion.

E.R. Cena is Senior Writer, UC Davis Department of Nutrition; and F.M. Steinberg is Professor and Department Chair, UC Davis Department of Nutrition. Robert M. Hackman, Research Nutritionist in the UC Davis Department of Nutrition, served as Guest Associate Editor for this article. This work was supported in part by the UC Davis Center for Health and Nutrition Research, established with funding from the State of California Vitamin Price Fixing Consumer Settlement Fund.

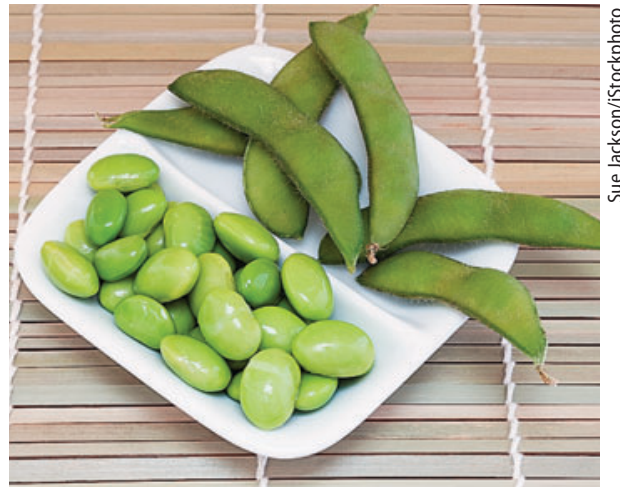

The beneficial effects of soy have been observed in Asian populations, which have lower heart disease rates than Western populations.

\section{References}

Ambra R, Rimbach G, de Pascual Teresa S, et al. 2006. Genistein affects the expression of genes involved in blood pressure regulation and angiogenesis in primary human endothelial cells. Nutr Metab Cardiovasc Dis 16(1):35-43

Anderson JW, Johnstone BM, Cook-Newell ME. 1995

Meta-analysis of the effects of soy protein intake on serum lipids. N Engl J Med 333(5):276-82

Arora A, Nair MG, Strasburg GM. 1998. Antioxidant activities of isoflavones and their biological metabolites in a liposomal system. Arch Biochem Biophys 356(2):133-41. Atkinson C, Frankenfeld CL, Lampe JW. 2005. Gut bacterial metabolism of the soy isoflavone daidzein: Exploring the relevance to human health. Exp Biol Med (Maywood) 230(3):155-70.

Atkinson C, Newton KM, Bowles EJ, et al. 2008. Demographic, anthropometric and lifestyle factors and dietary intakes in relation to daidzein-metabolizing phenotypes among premenopausal women in the United States. Am J Clin Nutr 87(3):679-87.

Azadbakht L, Kimiagar M, Mehrabi Y, et al. 2007. Soy inclusion in the diet improves features of the metabolic syndrome: A randomized crossover study in postmenopausal women. Am J Clin Nutr 85(3):735-41.

Beavers KM, Jonnalagadda SS, Messina MJ. 2009. Soy consumption, adhesion molecules and pro-inflammatory cytokines: A brief review of the literature. Nutr Rev 67(4):213-21.

Bonacasa B, Siow RCM, Mann GE. 2011. Impact of dietary soy isoflavones in pregnancy on fetal programming of endothelial function in offspring. Microcirculation 18(4):270-85.

Chacko BK, Chandler RT, D'Alessandro TL, et al. 2007. Anti-inflammatory effects of isoflavones are dependent on flow and human endothelial cell PPARgamma. J Nutr 137(2):351-6.

Chen J, Lin H, Hu M. 2003. Metabolism of flavonoids via enteric recycling: Role of intestinal disposition. J Pharmacol Exp Ther 304(3):1228-35.

Davis J, Higginbotham A, O'Connor T, et al. 2007. Soy protein and isoflavones influence adiposity and develop ment of metabolic syndrome in the obese male ZDF rat. Ann Nutr Metab 51(1):42-52.

Ford ES, Giles WH, Mokdad AH. 2004. Increasing prevalence of the metabolic syndrome among U.S. adults. Diabetes Care 27(10):2444-9.

Gottstein N, Ewins BA, Eccleston C, et al. 2003. Effect of genistein and daidzein on platelet aggregation and monocyte and endothelial function. Br J Nutr 89(5):607-16.
Heneman KM, Chang HC, Prior RL, Steinberg FM. 2007. Soy protein with and without isoflavones fails to substantially increase postprandial antioxidant capacity. J Nutr Biochem 18(1):46-53.

Heron M, Hoyert DL, Murphy SL, et al. 2009. Deaths: Final data for 2006. Natl Vital Stat Rep 57(14):1-134.

Ito A, Bebo BF Jr, Matejuk A, et al. 2001. Estrogen treatment down-regulates TNF-alpha production and reduces the severity of experimental autoimmune encephalomyelitis in cytokine knockout mice. J Immunol 167(1):542-52.

Lampe JW, Chang JL. 2007. Interindividual differences in phytochemical metabolism and disposition. Semin Cancer Biol 17(5):347-53.

Lampe JW, Karr SC, Hutchins AM, Slavin JL. 1998. Urinary equol excretion with a soy challenge: Influence of habitual diet. Proc Soc Exp Biol Med 217(3):335-9.

Lichtenstein AH, Appel $\sqcup$, Brands M, et al. 2006. Diet and lifestyle recommendations revision 2006: A scientific statement from the American Heart Association Nutrition Committee. Circulation 114(1):82-96.

Lloyd-Jones D, Adams R, Carnethon M, et al. 2009. Heart disease and stroke statistics - 2009 update: A report from the American Heart Association Statistics Committee and Stroke Statistics Subcommittee. Circulation 119(3):e21-181.

Mann GE, Bonacasa B, Ishii T, Siow RC. 2009. Targeting the redox sensitive Nrf2-Keap1 defense pathway in cardiovascular disease: Protection afforded by dietary isoflavones. Curr Opin Pharmacol 9(2):139-45.

Mitchell JH, Gardner PT, McPhail DB, et al. 1998. Antioxidant efficacy of phytoestrogens in chemical and biological model systems. Arch Biochem Biophys 360(1):142-8.

Mortensen A, Kulling SE, Schwartz H, et al. 2009. Analytical and compositional aspects of isoflavones in food and their biological effects. Mol Nutr Food Res 53(Suppl 2):S266-309.

Morton MS, Arisaka O, Miyake N, et al. 2002. Phytoestrogen concentrations in serum from Japanese men and women over forty years of age. J Nutr 132(10):3168-71. Nagarajan S. 2010. Mechanisms of anti-atherosclerotic functions of soy-based diets. J Nutr Biochem 21(4):255-60.

Peluso MR, Winters TA, Shanahan MF, Banz WJ. 2000. A cooperative interaction between soy protein and its isoflavone-enriched fraction lowers hepatic lipids in male obese Zucker rats and reduces blood platelet sensitivity in male Sprague-Dawley rats. J Nutr 130(9):2333-42.

Pipe EA, Gobert CP, Capes SE, et al. 2009. Soy protein reduces serum $\mathrm{LDL}$ cholesterol and the LDL cholesterol:HDL cholesterol and apolipoprotein B:apolipoprotein A-I ratios in adults with type 2 diabetes. J Nutr 139(9):1700-6.
Reynen DJ, Kamigaki AS, Pheatt N, Chaput LA. 2007. The Burden of Cardiovascular Disease in California: A Report of the California Heart Disease and Stroke Prevention Program. California Department of Public Health. Sacramento, CA. 64 p.

Rimbach G, Boesch-Saadatmandi C, Frank J, et al. 2008. Dietary isoflavones in the prevention of cardiovascular disease - a molecular perspective. Food Chem Toxicol 46(4):1308-19.

Ronis MJ, Chen Y, Badeaux J, Badger TM. 2009. Dietary soy protein isolate attenuates metabolic syndrome in rats via effects on PPAR, LXR and SREBP signaling. J Nutr 139(8):1431-8.

Rosenson RS. 2006. Low high-density lipoprotein cholesterol and cardiovascular disease: Risk reduction with statin therapy. Am Heart J 151(3):556-63.

Setchell KD, Brown NM, Desai PB, et al. 2003. Bioavailability, disposition and dose-response effects of soy isoflavones when consumed by healthy women at physiologically typical dietary intakes. J Nutr 133(4):1027-35. Steinberg FM, Guthrie NL, Villablanca AC, et al. 2003. Soy protein with isoflavones has favorable effects on endothelial function that are independent of lipid and antioxidant effects in healthy postmenopausal women. Am J Clin Nutr 78(1):123-30.

Steinberg FM, Murray MJ, Lewis RD, et al. 2011. Clinica outcomes of a 2-y soy isoflavone supplementation in menopausal women. Am J Clin Nutr 93(2):356-67. Taku K, Umegaki K, Sato Y, et al. 2007. Soy isoflavones lower serum total and LDL cholesterol in humans: A meta-analysis of 11 randomized controlled trials. Am J Clin Nutr 85(4):1148-56.

Tikkanen MJ, Wahala K, Ojala S, et al. 1998. Effect of soybean phytoestrogen intake on low density lipoprotein oxidation resistance. Proc Natl Acad Sci USA 95(6):3106-10.

Welty FK, Lee KS, Lew NS, Zhou JR. 2007. Effect of soy nuts on blood pressure and lipid levels in hypertensive, prehypertensive and normotensive postmenopausal women. Arch Intern Med 167(10):1060-7.

Wiseman H, O'Reilly JD, Adlercreutz H, et al. 2000. Isoflavone phytoestrogens consumed in soy decrease $F(2)$-isoprostane concentrations and increase resistance of low-density lipoprotein to oxidation in humans. Am J Clin Nutr 72(2):395-400.

Zhan S, Ho SC. 2005. Meta-analysis of the effects of soy protein containing isoflavones on the lipid profile. Am J Clin Nutr 81(2):397-408.

Zhang X, Shu XO, Gao YT, et al. 2003. Soy food consumption is associated with lower risk of coronary heart disease in Chinese women. J Nutr 133(9):2874-8. 